\title{
Multi-Trench Fibers: A Novel Approach for Rare Earth Doped Large-Mode-Area Fiber Lasers
}

\author{
Jayanta K. Sahu,* Deepak Jain, and Yongmin Jung \\ Optoelectronics research center, University of Southampton, UK \\ email: jks@orc.soton.ac.uk
}

\begin{abstract}
We review the progress towards multi-trench fibers for high power fiber laser applications. Such fiber designs address several challenges associated with power scaling of high power fiber laser.

OCIS codes: (060.2280) Fiber design and fabrication; (060.3510) Lasers, fibers
\end{abstract}

\section{Introduction}

Fiber lasers have become the backbone of high power laser sources over the last decade [1]. However, they are still unable to fulfill the demand of high power required for applications like next-generation particle accelerators, nuclear transmutation, nuclear waste treatment, astrophysics, and other industrial applications due to non-linear detrimental effects [2]. In order to avoid, non-linear effects, a large effective area $\left(A_{\text {eff }}\right)$ of the fundamental $(F M)$ is successfully employed by the fiber laser community. In order to exploit the large $A_{\text {eff }}$ of FM, different novel fiber designs have been proposed. Majority of the proposed large mode area (LMA) fiber designs have drawbacks, such as fabrication complexity and cost, fiber handling, i.e. cleaving and splicing. We tried to address these issues by proposing all-solid structure fibers having cylindrical symmetry, called multi-trench fiber (MTF) [3-4]. Cylindrical symmetrical fiber design ensures relatively simple fabrication and can be fabricated by conventional fiber fabrication technique, such as modified vapor deposition process (MCVD). Moreover, LMA fiber designs, such as PCF, 2D-ASPBGF, and LCF etc. require core refractive index to be same as of cladding. Furthermore, in the case of active fiber core needs to be doped with Rare-earth ions with additional co-dopants, such as aluminium (Al) and phosphorous (P). Unfortunately, rare-earth elements (such as Yb, Er, Tm, and Ho) and co-dopants (Al, P) used for high power fiber laser are index raising components. In order to match the refractive index of core and cladding, one needs to dope the core with index decreasing components such as fluorine (F) or Boron (B), which is a cumbersome process to implement. We recently demonstrated single-trench fiber (STF) which can afford core refractive index to be higher than cladding index on the order of $\sim 5 \times 10^{-4}[5-6]$. Moreover, our optimized MCVD process in conjunction with solution doping technique can very well control the doped core refractive index to be $\sim 5 \times 10^{-4}$. Thus, STF dramatically reduces the cost involved in LMA fiber fabrication, hence suitable for mass production. In this paper, we review progress towards MTF and STF for high power fiber laser applications.

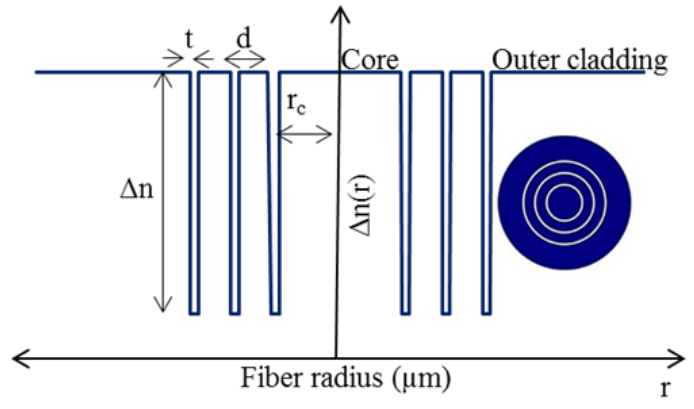

(a)

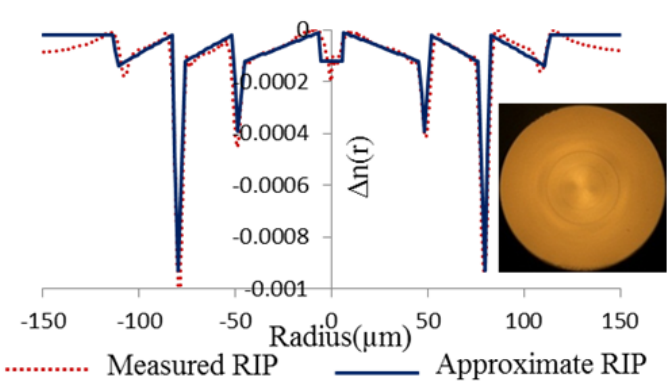

(b)

Fig. 1 (a) Schematic of refractive index profile of MTF. Inset shows the 2-D cross-section of MTF. Where $r_{c}$ is core radius, $t$ is trench thickness, $\mathrm{d}$ is resonant thickness, and $\Delta \mathrm{n}$ is the index difference between cladding and trench (b) The fabricated refractive index profile of $90 \mu \mathrm{m}$ core MTF for rod type configuration. Inset shows the microscope image of fiber $2 \mathrm{D}$ cross-section.

\section{Multi trench fiber for rod-type configuration}

Figure 1(a) shows the schematic of MTF. Inset shows the 2D-cross-section of fiber. MTF ensures high leakage losses to the higher order modes $(\mathrm{HOMs})$ of core by inducing resonant coupling between core and resonant ring modes. Numerical analysis using finite element method (FEM) ensures an $\mathrm{A}_{\mathrm{eff}}$ of larger than $5,000 \mu \mathrm{m}^{2}$ in 
a $100 \mu \mathrm{m}$ core MTF while ensuring very high leakage loss of HOMs (higher than 90dB/m) and low loss of FM (lower than $1 \mathrm{~dB} / \mathrm{m}$ ) for optimum cladding parameters. Figure 1(b) shows the measured refractive index profile of a $90 \mu \mathrm{m}$ core fabricated MTF. Inset shows the microscope image of 2-D cross section of fiber. In this first iteration of fabrication, we could not hit all the optimum fiber design parameters. Numerical simulation over the measured RIP shows FM mode loss is lower than $0.05 \mathrm{~dB} / \mathrm{m}, \mathrm{LP}_{11}$ mode loss is larger than $12 \mathrm{~dB} / \mathrm{m}$, and other HOMs loss are larger $35 \mathrm{~dB} / \mathrm{m}$. On the other hand, their power fractions in core are larger than $97 \%$ for FM, lower than $60 \%$ for $\mathrm{LP}_{11}$, and lower than $76 \%$ for other HOMs. The $\mathrm{A}_{\text {eff }}$ is larger than $3,100 \mu \mathrm{m}^{2}$. Figure 2(a) shows the $\mathrm{S}^{2}$ measurement in a $90 \mathrm{~cm}$ long fiber [5]. We did not observe any signature of the HOMs. We also did qualitative analysis of output beam using experimental set-up as shown in Fig. 2(b). Figure 2(b) shows the output beam profile captured by the CCD camera. The output beam profile is nearly Gaussian although a few cladding modes can also be seen. This fiber shows significant microbend sensitivity as the outer diameter of the current fiber is merely $370 \mu \mathrm{m}$. To overcome this, the outer diameter of fiber should have been $\sim 1 \mathrm{~mm}$. This microbend sensitivity makes it significantly difficult to eliminate the cladding and ring modes, and thus difficult to precisely calculate the loss of the FM mode.

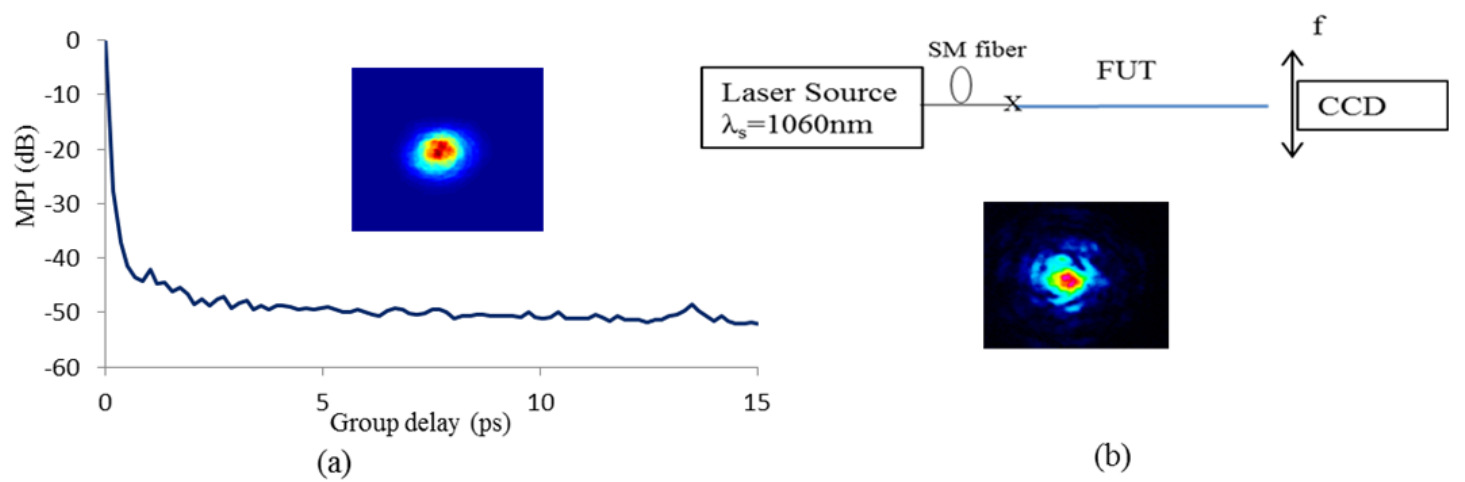

Fig. 2 (a) MPI versus group delay for 90cm long rod-type fiber. Inset shows the obtained FM image from $\mathrm{S}^{2}$ measurement. (b) Output image captured by CCD camera using the experimental set up shown here.

\section{Multi trench fiber for bend configuration}

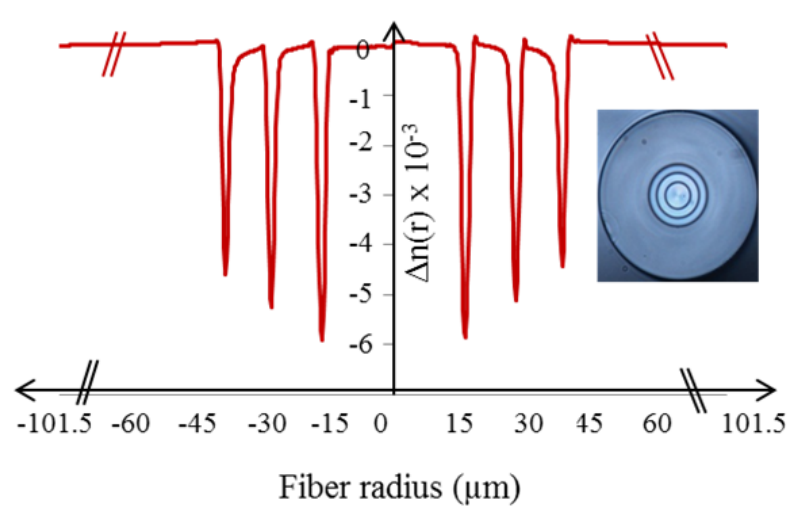

(a)

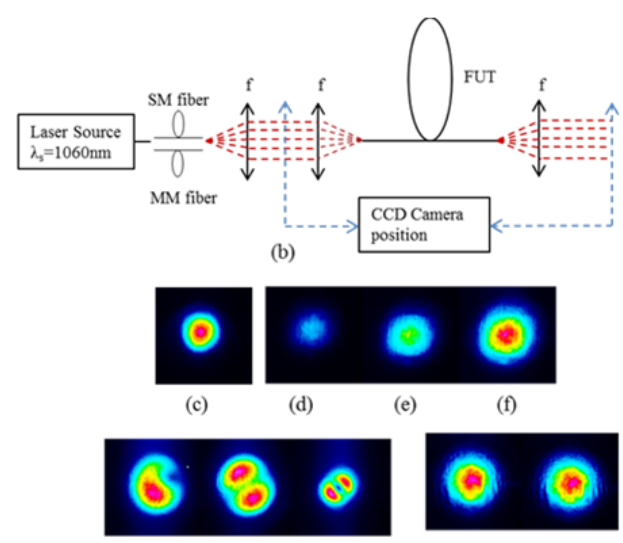

(h)

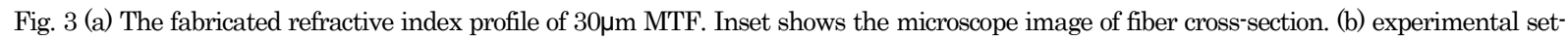
up used for single-mode verification (c) the output profile for optimum input launching (d-f) output profile for offset input launching conditions. (g) various input launch modes (h) output profiles with respect to multimode input launch. 
Figure 3 (a) shows the refractive index profile of the fabricated $30 \mu \mathrm{m}$ MTF for bend configuration. Numerical simulations show that a $30 \mu \mathrm{m}$ core diameter MTF with $\mathrm{d}=8 \mu \mathrm{m}, \mathrm{t}=2 \mu \mathrm{m}$, and $\Delta \mathrm{n}=-0.005$, provides an effective area $\left(\mathrm{A}_{\text {eff }}\right)$ of the FM larger than $407 \mu \mathrm{m}^{2}$ in $28-50 \mathrm{~cm}$ bend diameter range at wavelength $1060 \mathrm{~nm}$. Figure 3(b) shows the experimental set-up used to verify the single mode behaviour of fiber. For various off-set and multimode launching, fiber ensures a single mode operation. We also compared fibers with high and low-index coating to study the impact of polymer coating on single-mode behaviour. $S^{2}$ measurement ensures effective single-mode behaviour in both high and low-index polymer coated fibers. Although, MTF offers effective single mode operation with very high suppression of the HOMs but they still require core refractive index matched to the cladding index. On the hand, we recently proposed STF which can offer core refractive index to be higher than cladding.

\section{Single-trench fiber for record delocalization}

Figure 4(a) shows the schematic of STF having higher refractive index of core than cladding. Figure 4(b) shows the refractive index of fabricated Yb-dopants doped preform. D-shaped fiber was drawn with outer diameter of $185 \mu \mathrm{m}$ and core diameter of $30 \mu \mathrm{m}$. Fiber was coated with low index polymer. Figure 4(c) and 4(d) shows the output beam profile of fiber with respect to multimode input beam launch using set-up as shown in Fig. 3(b). These measurements ensure an effective single mode operation. This fiber shows more than $80 \%$ slope efficiency at wavelength $1040 \mathrm{~nm}$ in a $4 \%-4 \%$ laser cavity. Also, numerical simulations show that the effective single mode operation can be ensured by high loss of HOMs (higher than $10 \mathrm{~dB} / \mathrm{m}$ ) and high power delocalization of HOMs ( $30 \%$ discrimination between HOMs and FM) in a core of $50 \mu \mathrm{m}$. An $\mathrm{A}_{\text {eff }}$ larger than $1,000 \mu \mathrm{m}^{2}$ can be achieved at $20 \mathrm{~cm}$ bend radius.

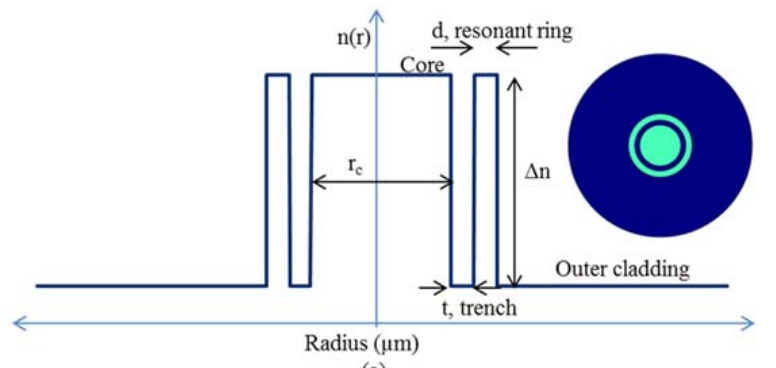

(a)

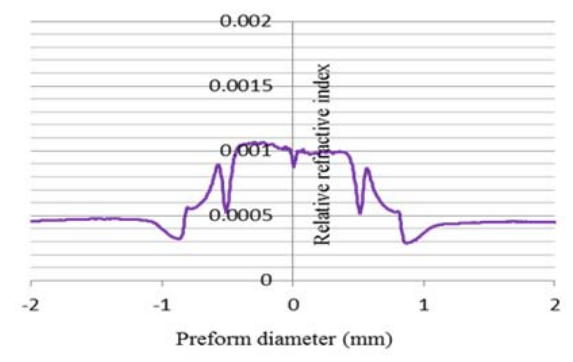

(b)

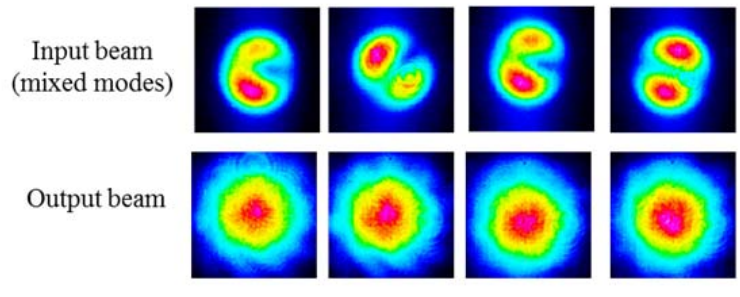

(c)

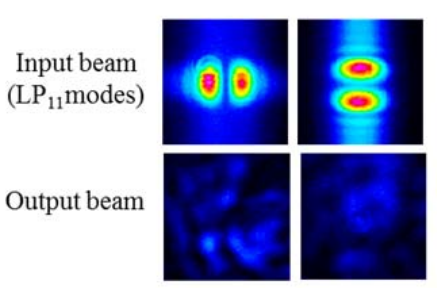

(d)

Fig. 4 (a) Schematic of refractive index profile of STF. Inset shows the 2-D cross-section of fiber. (b) refractive index profile of fabricated Ybdoped preform (c) and (d)output beam profiles with respect to mixed mode $\left(\mathrm{LP}_{01}+\mathrm{LP}_{11}\right)$ and $\mathrm{LP}_{11}$ input beam launch.

\section{Conclusion}

We have demonstrated novel fiber designs which offer large $\mathrm{A}_{\text {eff }}$ with effective SM operation by exploiting high loss and delocalization of HOMs. Proposed designs can achieve $A_{\text {eff }}$ larger than $1,000 \mu \mathrm{m}^{2}$ and $10,000 \mu \mathrm{m}^{2}$ in a compact and rod-type fiber laser respectively. Being a cylindrical and all-solid structure, the proposed fibers are suitable for mass production. Furthermore, unlike PCFs, these fibers are easy to cleave and splice.

\footnotetext{
4. References

[1] C. Jauregui et. al., "High-power fiber lasers," Review, Nature Photonics, vol. 7, 861-867, 2013.

[2] G. P. Agrawal et. al., "Nonlinear Fiber optics", $4^{\text {th }}$ edition, Academic Press, 2007.

[3] D. Jain, et. al., "Mode area scaling with Multi-trench rod-type fibers," Opt. Exp. 21, 1448-1455, 2013.

[4] D. Jain et. al., "Bending performance of large mode area multi-trench fibers," Opt. Exp. 21, 26663-26670, 2013.

[5] D. Jain et. al., "Large mode area multi-trench fiber with delocalization of higher order modes," Invited, IEEE JSTQE 20, $0902909,2014$.

[6] D. Jain et. al., "First demonstration of single trench fiber for delocalization of higher order modes," Invited, SF1N.1, CLEO, San Jose, 2014.
} 